\title{
LA PÉRIODISATION DES MATHÉMATIQUES CLASSIQUES *
}

La dichotomie " médiéval - moderne " a dominé, et domine encore, l'historiographie des mathématiques. Elle s'offre comme l'instrument indispensable au découpage des périodes, et ainsi comme l'un des pivots de la constitution de la diachronie des mathématiques, tout au moins avant le milieu du XVII siècle. On comprend dès lors que, pour toutes les parties engagées dans le fameux débat, encore vivace, sur les concepts et les méthodes de l'histoire des sciences, ce soit une donnée inéluctable, bien plus, un postulat préalable à tout découpage historique des mathématiques. Il suffit pour s'en convaincre de consulter les sommes historiques d'un Montucla ou d'un Cantor, les synthèses, plus récentes, comme celle de Bourbaki, les monographies plus spécialisées, telles celles de Zeuthen hier ou de Youschkevitch aujourd'hui. Tous admettent ce postulat, pour saisir, comme ils aiment à le dire, l'avènement des matnématiques modernes, et s'accordent pour introduire une notion supplémentaire, celle de la Renaissance mathématique, qui soulève bien plus de problèmes qu'elle n'en résout. Rien donc de plus naturel que d'examiner cette dichotomie " médiéval-moderne " à la lumière des progrès accomplis, au cours de ces dernières décennies, dans notre connaissance des mathématiques en arabe entre le IXcet le $\mathrm{XVI}^{e}$ siècle, et dans l'étude du monde latin postérieur au XII" siècle ${ }^{1}$. Mais arrêtons-nous quelque peu encore sur cette dichotomie elle-même.

Dans les écrits des historiens des mathématiques, cette fameuse opposition entre " médiéval » et « moderne » n'est pas seulement destinée à

\footnotetext{
* Une première version, plus courte, de cet article, a été lue au Colloque consacré à la mémoire d'Alexandre Koyré, qui s'est tenu au Collège de France en juin 1986.

1. Cf. Marshall Clagett, Archimedes in the Middle Ages, t. IV, Philadelphia, American philosophical society, 1980 .
}

Revue de synthèse : IVe S. N ${ }^{\text {ou }} 3-4$, juil.-déc. 1987. 
jalonner les étapes d'une chronologie : intentionnellement ou non, les auteurs l'invoquent afin de désigner deux positivités mathématiques distinctes. Peu importe alors que les " mathématiques modernes" soient représentées comme un dépassement radical des mathématiques médiévales, ou comme un développement naturel de celles-ci, ou enfin dans la continuité directe des mathématiques hellénistiques, en sautant pieds joints par-dessus les mathématiques médiévales. Ces attitudes au premier abord opposées et exclusives s'accordent cependant pour admettre que les mathématiques médiévales non seulement constituent une entité historique, mais que celle-ci s'oppose à une autre entité, formée à la Renaissance. Mais, quelque parti que l'on adopte, on ne tardera pas à se trouver confrontré à des difficultés majeures. La première surgit des sens du mot " médiéval "; a-t-on, en effet, le droit de désigner, sous ce même mot, les mathématiques latines, aussi bien que byzantines ou arabes, pour ne retenir qu'elles ? Est-il légitime de les regrouper, en leur associant parfois, comme le font d'éminents historiens, les mathématiques chinoises et indiennes, sous prétexte qu'elles sont contemporaines ? Il n'est du reste pas rare que l'historien, sans formuler explicitement cette question, commence pourtant par l'esquiver en retraçant tour à tour l'histoire de ces trois - ou cinq - mathématiques. Mais, comme alors il ne peut, ni en droit ni en fait, supposer un trait génériquement commun aux mathématiques médiévales, la dichotomie s'effondre d'elle-même, et, avec elle, le découpage proposé.

Bien plus redoutable est la question que suscite l'emploi du terme " médiéval » lorsqu'il s'agit de la même mathématique : on cherchera en vain les raisons qui justifient cet usage pour Léonard de Pise au XIII siècle et non point pour Luca Pacioli à la fin du XVe siècle, par exemple ; ou pour al-Karaji à la fin du Xe siècle et non point pour al-Yazadi au début du XVIIe siècle.

Apparemment d'ordre méthodologique, de telles questions s'avèrent donc à la fois historiques et épistémologiques ; et, pour mieux éclairer cette dichotomie, il nous faut connaître plus sûrement les composantes des mathématiques médiévales, et appréhender, dans chaque cas, les traits essentiels. Ce sont précisément certains de ces aspects que je me propose d'esquisser ici, dans le seul cas des mathématiques écrites en arabe entre le IX'e et le XVII' siècle.

Revenons donc à Bagdad au début du IXe siècle. Le mouvement de traduction des grandes compositions mathématiques hellénistiques est à son apogée. Deux caractères de cette entreprise, insuffisamment soulignés, sont pourtant de première importance : les traductions sont conduites par des mathématiciens, souvent de premier ordre, comme Thäbit ibn Qurra, par exemple ; elles sont suscitées par la recherche la plus avancée de l'épo- 
que. Tout indique ainsi que la traduction par Qusțā ibn Lūqã des Arithmétiques de Diophante, vers 870 , fut motivée par une recherche, déjà existante, sur l'analyse indéterminée ou l'analyse diophantienne rationnelle. De même les traductions des Miroirs ardents de Dioclès ou d'Anthémios de Tralles devaient satisfaire les besoins de la recherche en ce domaine. On peut ainsi multiplier les exemples qui tous manifestent la liaison étroite entre la traduction, d'une part, la recherche et l'innovation, de l'autre. Manquer cet aspect, c'est s'interdire de comprendre les propriétés de cette traduction, ainsi que la diffusion du savoir traduit et les voies et les raisons des innovations.

En un mot, la traduction représente un grand moment d'expansion en arabe des mathématiques hellénistiques. Or, c'est précisément à cette période, et dans ce milieu - celui de l'Académie (Maison de la Sagesse) de Bagdad - qu'al-Khwärizmi rédige un livre dont le sujet, aussi bien que le style, sont nouveaux. Dans ces pages, on voit pour la première fois surgir l'algèbre comme discipline mathématique, distincte et indépendante. L'événement fut crucial, et perçu comme tel par les contemporains, tant pour le style de cette mathématique que pour l'ontologie de son objet, et, surtout, la richesse des possibilités qu'elle offrait désormais. Le style, en effet, est à la fois algorithmique et démonstratif. Il a, en outre, fallu concevoir un être mathématique suffisamment général pour recevoir des déterminations de genres différents, mais doué, d'autre part, d'une existence indépendante de ses propres déterminations. Chez al-Khwärizmĩ déjà (environ 830), l'objet algébrique renvoie aussi bien à un nombre rationnel qu'à une quantité irrationnelle ou à une grandeur géométrique. La science elle-même, l'algèbre, doit être à la fois apodictique et appliquée. Or cette nouvelle ontologie, ainsi que la combinaison des deux démarches, démonstrative et appliquée, ont frappé les philosophes de l'époque. Sans nous étendre sur ces aspects, observons simplement que d'ores et déjà, avec cette algèbre, on entrevoit l'immense potentialité dont disposeront les mathématiques à partir du IXe siècle : l'application des disciplines mathématiques les unes aux autres. En d'autres termes, si l'algèbre, en raison de la généralité de son objet et de la nouvelle ontologie ainsi introduite, a rendu possibles ces applications, celles-ci à leur tour, par leur nombre et la diversité de leur nature, ne cesseront de modifier la configuration des mathématiques après le IX' siècle.

Les successeurs d'al-Khwärizmī entreprennent progressivement l'application de l'arithmétique à l'algèbre, de l'algèbre à l'arithmétique, de l'une et de l'autre à la trigonométrie, de l'algèbre à la théorie euclidienne des nombres, de l'algèbre à la géométrie, de la géométrie à l'algèbre. Ces applications furent toujours les actes fondateurs de nouvelles disciplines, ou tout au moins de nouveaux chapitres. C'est ainsi que se consti- 
tueront l'algèbre des polynômes, l'analyse combinatoire, l'analyse numérique, la résolution numérique des équations, la nouvelle théorie élémentaire des nombres, la construction géométrique des équations. À cela, nous devrions ajouter d'autres conséquences, telle la séparation de l'analyse diophantienne entière de l'analyse diophantienne rationnelle, devenue un chapitre à part entière de l'algèbre sous le titre de l' " analyse indéterminée ".

De telles applications expriment en réalité la double dialectique qui domine toute la production mathématique au $\mathrm{X}^{\mathrm{c}}$ siècle et au cours des sept siècles suivants : la dialectique entre l'algèbre et l'arithmétique, et la dialectique entre l'algèbre et la géométrie. C'est dire que, pour comprendre la majeure partie de l'activité mathématique pendant sept siècles au moins, il faut saisir le rôle capital de la science nouvelle, l'algèbre, dans la naissance de cette dialectique, et ainsi se placer au cœur de ce mouvement de réorganisation et de restructuration de ces disciplines l'une par l'autre. L'application de l'arithmétique à l'algèbre — « l'arithmétisation de l'algèbre ", comme je l'ai nommée naguère - avait abouti à la fin du Xe siècle, chez le mathématicien de Bagdad, al-Karajì, à la constitution de l'algèbre des polynômes. À partir d'al-Karajī, et jusqu'au XVIII' siècle, un traité d'algèbre s'organise autour des opérations arithmétiques sur les polynômes. Il suffit pour illustrer cette nouvelle situation de rappeler ici les principaux chapitres d'un traité d'algèbre, et quelques-uns des résultats qu'il renferme. Prenons le traité du mathématicien du XII' siècle, al-Samaw'al : l'auteur commence par définir en toute généralité la notion de puissance algébrique et, grâce à la définition $\mathrm{x}^{\circ}=1$, donne la règle équivalente à $\mathrm{x}^{\mathrm{m}} \mathrm{x}^{\mathrm{n}}=\mathrm{x}^{\mathrm{m}+\mathrm{n}}, \mathrm{m}, \mathrm{n} \epsilon \mathbf{Z}$. Vient ensuite l'étude des opérations arithmétiques sur les monômes et les polynômes, et notamment celle de la divisibilité des polynômes ainsi que celle de l'approximation des fractions entières par les éléments de l'anneau des polynômes. Pour être en mesure d'entreprendre ces recherches sur les polynômes, on consacre un chapitre à l'établissement de la formule du développement binomial et le tableau des coefficients. C'est à l'occasion de la démonstration de cette formule qu'apparait, sous une forme archaïque certes, l'induction complète finie comme procédé de preuve.

Vient ensuite la réponse à la question que voici : " comment la multiplication, la division, l'addition, la soustraction et l'extraction des racines peuvent-elles être utilisées pour des quantités irrationnelles ? " La réponse à cette question a constitué la première étude - empirique il est vrai - des extensions algébriques finies du corps des rationnels. On trouve également un important chapitre sur l'analyse indéterminée ou l'analyse diophantienne rationnelle. Or ce livre d'al-Samaw'al, comme celui d'alKaraji auparavant, et comme les livres des mathématiciens de cette tra- 
dition de l'algèbre arithmétique, comporte un petit chapitre sur les équations algébriques, constitué par la solution de l'équation quadratique. Ce chapitre sur les équations algébriques, non seulement est d'un volume réduit, mais n'occupe pas non plus une place centrale. Autrement dit, alors qu'il représentait la partie principale du livre d'al-Khwãrizmi, et qu'il occupera toute la place - on le verra - dans la tradition géométrique, il apparaît ici comme un petit chapitre parmi d'autres. Dans certains traités de la même tradition, ce chapitre comporte en outre une recherche pour la résolution algébrique de l'équation cubique ${ }^{2}$. Il est clair en tout cas que les algébristes-arithméticiens s'occupaient intentionnellement beaucoup plus de la constitution du calcul algébrique abstrait que de la théorie des équations algébriques. Il est vrai que les travaux de ces mathématiciens ont mieux fait connaître la structure algébrique du corps des nombres réels, ou plus précisément des extensions algébriques finies du corps des rationnels.

Les résultats obtenus, ainsi que les théorèmes démontrés par les algébristes des $\mathrm{XI}^{e}$-XII ${ }^{e}$ siècles, ont été, pour la plupart, attribués par les historiens aux mathématiciens des XVIe-XVII' siècles.

En revanche, l'application de l'algèbre à l'arithmétique traditionnelle a donné naissance à plusieurs chapitres tels que l'analyse numérique, où l'on trouve différentes méthodes pour l'extraction de la racine $\mathrm{n}^{\text {ième }} \mathrm{d}$ 'un entier, ainsi que de multiples méthodes d'approximation ; ou encore la résolution numérique des équations algébriques, et la théorie classique des nombres. Pour illustrer cette application, considérons, très brièvement, le dernier exemple, celui de la théorie classique des nombres.

À la fin du IX' siècle, on avait traduit les textes arithmétiques grecs les plus importants, notamment les Livres arithmétiques d'Euclide et l'Introduction arithmétique de Nicomaque de Gérase. Mais, alors qu'Euclide donne une théorie des nombres parfaits, ni lui, ni Nicomaque, ni du reste aucun auteur grec n'avait fait la théorie des nombres amiables. Thābit ibn Qurra, traducteur du livre de Nicomaque, réviseur d'une traduction des Éléments, et lui-même éminent mathématicien, décide d'élaborer cette théorie. Il énonce et démontre le théorème le plus important jusqu'ici pour ces nombres, et aujourd'hui connu sous son nom :

Pour $\mathrm{p}>1$, soit $\mathrm{p}_{\mathrm{n}}=3.2^{\mathrm{n}}-1, \mathrm{q}_{\mathrm{n}}=9.2^{2 \mathrm{n}-1}-1$; si $\mathrm{p}_{\mathrm{n}-1}, \mathrm{p}_{\mathrm{n}}$, $\mathrm{q}_{\mathrm{n}}$ sont premiers, alors $\mathrm{a}=2^{\mathrm{n}} \mathrm{p}_{\mathrm{n}-1} \mathrm{p}_{\mathrm{n}}$ et $\mathrm{b}=2^{\mathrm{n}} \mathrm{q}_{\mathrm{n}}$ sont amiables, $\mathrm{a}$ est abondant et $\mathrm{b}$ est déficient.

2. Cf. Roshdi RASHED, Entre arithmétique et algèbre. Recherches sur l'histoire des mathématiques arabes, Paris, Les Belles Lettres, 1984, p. 28-29. 
Ibn Qurra donne 9 lemmes, en deux groupes, pour prouver ce théorème. Les lemmes du premier groupe traitent, en fait, de la détermination des parties aliquotes d'un entier naturel. Le deuxième groupe de lemmes porte plus spécifiquement sur la formation des nombres parfaits, abondants, et déficients. Pour engendrer ces nombres Ibn Qurra prouve, par exemple, le théorème suivant ${ }^{3}$ :

$$
\text { Soit } \mathrm{s}=\stackrel{\mathrm{k}}{\Sigma}=\mathrm{o}_{\mathrm{n}}^{\mathrm{k}} \text {, et } \mathrm{p} \neq 2 \text { un nombre premier, alors }
$$

(1) $\sigma_{\circ}\left(2^{\mathrm{n}} \mathrm{s}\right)=2^{\mathrm{n}} \mathrm{s}$ si s est un nombre premier,

(2) $\sigma_{\circ}\left(2^{\mathrm{n}} \mathrm{p}\right)>2^{\mathrm{n}} \mathrm{p}$ si $\mathrm{p}<\mathrm{s}$,

(3) $\sigma_{\circ}\left(2^{\mathrm{n}} \mathrm{p}\right)<2^{\mathrm{n}} \mathrm{p}$ si $\mathrm{p}>\mathrm{s}$, $\left|\sigma_{\circ}\left(2^{\mathrm{n}} \mathrm{p}\right)-2^{\mathrm{n}} \mathrm{p}\right|=|\mathrm{s}-| \mathrm{p}$.

Il est clair que (1) - que nous trouvons dans les Éléments d'Euclide - a pour fonction d'engendrer les nombres parfaits, (2) et (3) les nombres abondants et déficients respectivement.

La nouvelle théorie d'Ibn Qurra demeure euclidienne : les concepts et les méthodes de la preuve sont euclidiens. Ce qui signifie que, immédiatement après la traduction d'Euclide et de Nicomaque, le premier acte fut l'extension du domaine de l'arithmétique grecque dans cette nouvelle théorie, mais en pur style euclidien.

Un algébriste du XIII siècle, de la tradition d'al-Karaji, al-Fārisi, a repris le problème et le théorème d'Ibn Qurra pour en donner une démonstration algébrique. Il a été ainsi amené à définir et à étudier les deux premières fonctions arithmétiques : la fonction « somme des diviseurs d'un entier ", et la fonction « nombre des diviseurs d'un entier ». Il commence par énoncer et démontrer les propositions suivantes :

(1) Si $\mathrm{n}=\mathrm{p}_{1} \mathrm{p}_{2}$, avec $\mathrm{p}_{2}$ premier et $\left(\mathrm{p}_{1}, \mathrm{p}_{2}\right)=1$, alors

$$
\sigma_{\circ}(\mathrm{n})=\mathrm{p}_{2} \sigma_{\circ}\left(\mathrm{p}_{1}\right)+\sigma\left(\mathrm{p}_{1}\right) \text {, }
$$

(2) Si $\mathrm{n}=\mathrm{p}^{\mathrm{r}}$, $\mathrm{p}$ premier, alors

$$
\sigma_{\circ}(n)={ }_{k=0}^{r} p^{-1} p^{k}=\frac{p^{r}-1}{p-1}
$$

(3) Si $\mathrm{n}=\mathrm{p}_{1} \mathrm{p}_{2},\left(\mathrm{p}_{1}, \mathrm{p}_{2}\right)=1$, alors

$$
\sigma_{\circ}(\mathrm{n})=\mathrm{p}_{1} \sigma_{\circ}\left(\mathrm{p}_{2}\right)+\mathrm{p}_{2} \sigma_{\circ}\left(\mathrm{p}_{1}\right)+\sigma_{\circ}\left(\mathrm{p}_{1}\right) \sigma_{\circ}\left(\mathrm{p}_{2}\right)
$$

on voit immédiatement qu'il connaît l'expression :

$$
\sigma\left(p_{1} p_{2}\right)=\sigma\left(p_{1}\right) \sigma\left(p_{2}\right)
$$

et la propriété fondamentale de la fonction $\sigma$ : elle est multiplicative.

Toutes ces propositions sont attribuées à Descartes ; bien plus, elles

3. Notons par $\sigma .(n)$ la somme des diviseurs propres de l'entier $n$, et par $\sigma(n)=\sigma .(n)$ +1 la somme de ses diviseurs. 
représentent sa contribution la plus remarquable à la théorie des nombres. Mais, plus importante que le problème d'attribution est la méthode qu'applique al-Färisi pour démontrer ces propositions : il fait explicitement appel à la méthode combinatoire et algébrique. Cette même méthode lui permet d'avancer et de démontrer les propositions suivantes concernant la fonction (n), nombre des diviseurs propres d'un entier $n$, et (n), nombre des diviseurs de $\mathrm{n}$ :

(1) Si $\mathrm{n}=\mathrm{p}_{1} \mathrm{p}_{2} \ldots \mathrm{p}_{\mathrm{r}}$, avec $\mathrm{p}_{1}, \mathrm{p}_{2}, \ldots, \mathrm{p}_{\mathrm{r}}$ nombres premiers distincts, alors $\tau_{0}(n)=1+\left(\begin{array}{l}r \\ 1\end{array}\right)+\ldots+\left(r \frac{r}{p}\right)$.

(2) Si $\mathrm{n}=\mathrm{p}_{1}^{\mathrm{e}_{1}} \mathrm{p}_{2}{ }^{\mathrm{e}} \ldots \mathrm{p}_{\mathrm{r}}^{\mathrm{e}}$, avec $\mathrm{p}_{1}, \mathrm{p}_{2}, \ldots, \mathrm{p}_{\mathrm{r}}$ nombres premiers distincts, alors

$$
\tau(n)={ }_{i} \prod_{=1}^{r}\left(e_{i}+1\right) \text {. }
$$

Ces deux propositions ont été ensuite retrouvées respectivement par l'abbé Deidier (XVIII siècle) et Montmort.

On voit donc que l'application de l'algèbre à l'arithmétique euclidienne a abouti à l'étude des fonctions arithmétiques et, plus généralement, à l'étude algébrique des diviseurs propres. Cette tendance est encore plus manifeste lorsque le même al-Fārisĩ examine les nombres figurés et en donne une interprétation combinatoire identique à celle que l'on trouvera chez Frénicle, Pascal et Bernoulli ${ }^{4}$.

Ainsi, à partir du IXe siècle, le paysage mathématique n'est plus le même : il se transforme et ses horizons reculent. Tout d'abord, on assiste à l'extension de l'arithmétique et de la géométrie hellénistiques. Théorie des coniques, théorie des parallèles, théorie euclidienne des nombres, méthodes archimédiennes pour la mesure des aires et des volumes, problèmes isopérimétriques : tous ces domaines deviennent objet d'étude pour les mathématiciens les plus prestigieux - Thäbit ibn Qurra, al-Qühi, Ibn al-Haytham, entre autres - qui parviennent, par de profondes recherches, à les développer dans le même style que leurs devanciers. D'autre part, au sein de ces mathématiques hellénistiques elles-mêmes, on aménage des régions non hellénistiques : c'est grâce aux méthodes algébriques, en effet, qu'on a pu étudier, comme on vient de le voir, les premières fonctions arithmétiques, ainsi que les séries des nombres figurés, en promouvant un nouveau secteur dans la théorie euclidienne des nombres. Si, enfin, on ajoute à cette extension sur le même terrain et à la création de nouvelles régions les disciplines qui viennent d'être fondées et que nous avons déjà évoquées, on voit se dessiner le nouveau paysage

4. Cf. R. RASHED, op. cit. supra n. 2, p. 287 sqq. 
mathématique. Une analyse plus détaillée permettra de montrer que les relations entre les anciennes disciplines ne sont plus les mêmes, et que bien d'autres groupements se sont opérés. Ce changement de rapports est capital si l'on veut comprendre l'histoire des mathématiques. Prenons à titre d'exemple le livre $\mathrm{X}$ des Éléments. Ouvrage de géométrie pour Euclide, Pappus, ou même Ibn al-Haytham, il devient à partir de la fin du $X^{e}$ siècle un livre d'algèbre qui traite, si l'on use d'un autre langage, des extensions algébriques finies du corps des rationnels.

Tout aussi importante que cette modification du paysage mathématique est l'introduction d'un nouveau type de démonstration, inconnu auparavant, la démonstration algébrique. Même si, en effet, la démonstration géométrique de type euclidien ou archimédien demeure dominante, la démonstration algébrique s'impose dans plusieurs chapitres, comme l'algèbre des polynômes, l'analyse combinatoire, la nouvelle théorie des nombres. C'est d'ailleurs à cette seule démonstration que l'on recourait pour justifier les algorithmes de résolution algébrique ou numérique des équations.

D'autres techniques de grande importance ont vu le jour en cette même période ; l'examen de l'analyse locale, par exemple, nous permettra de mieux caractériser cette mathématique. Pour comprendre l'émergence du point de vue local, revenons à cette dialectique entre algèbre et géométrie que nous avons évoquée auparavant.

Sans justification théorique aucune, les mathématiciens du $X^{*}$ siècle amorcent une double traduction, jamais pensée auparavant : ils traduisent dans la langue de l'algèbre des problèmes solides, inconstructibles à la règle et au compas - la trisection de l'angle, les deux moyennes, l'heptagone régulier, notamment. C'est à cette même époque que certains mathématiciens et astronomes ont procédé par cette traduction algébrique pour déterminer les cordes de certains angles, afin de former la table des sinus. Les noms d'al-Māhāni, al-Khảzin, al-Birūni, jalonnent, entre autres, ce courant de traduction algébrique.

D'autre part, confrontés à la difficulté de résoudre par radicaux l'équation cubique, les algébristes, mais aussi géomètres, comme Abū al-Jüd b. al-Leith, ont été amenés à traduire cette équation dans la langue de la géométrie. Ils se trouvaient ainsi en mesure d'appliquer à l'étude de cette équation une technique, d'emploi alors assez courant dans l'examen des problèmes solides, c'est-à-dire l'intersection des courbes. Cette technique était d'usage fréquent dans les mathématiques grecques déjà, et parfaitement maîtrisée, comme l'attestent les travaux d'al-Qūhì et d'Ibn al-Haytham, par exemple.

C'est avec al-Khayyām (1048-1131, environ) que la première tentative pour fonder cette double traduction voit le jour. Celui-ci entend dépas- 
ser la recherche particulière, c'est-à-dire une recherche liée à telle ou telle forme de l'équation cubique, pour élaborer une théorie des équations et proposer, du même coup, un nouveau modèle de rédaction. La nouvelle théorie est celle des équations algébriques de degré $\leqslant 3$. Il étudie alors les équations du troisième degré à l'aide de courbes coniques, afin de déterminer les racines réelles positives. Pour élaborer cette nouvelle théorie, al-Khayyãm s'est trouvé contraint à mieux concevoir, et ainsi à formuler, les nouveaux rapports entre l'algèbre et la géométrie. Rappelons qu'à cet égard le concept fondamental introduit par al-Khayyām est celui d'unité de mesure qui, convenablement défini en rapport avec celui de dimension, permet l'application de la géométrie à l'algèbre.

La théorie des équations semble désormais, quoique timidement encore, relativement transcender le clivage entre l'algèbre et la géométrie. Rien n'empêche les auteurs de lui consacrer des mémoires en propre, et rien ne leur interdit de restreindre délibérément leur exposé à cette seule théorie. Contrairement aux algébristes arithméticiens, al-Khayyām écarte de son étude les chapitres qui occupaient une place centrale dans tout traité algébrique de l'époque, tels que l'étude des puissances algébriques, des polynômes, des opérations qu'on applique à ces derniers, l'étude des irrationnels algébriques, etc.

Dans son Traité, al-Khayyàm parvient à deux résultats remarquables que les historiens ont coutume d'attribuer à Descartes : une solution générale de toutes les équations du troisième degré par l'intersection de deux coniques ; et, d'autre part, un calcul géométrique rendu possible par le choix d'une longueur unité. Cependant, tout comme ses devanciers, alKhayyãm ne considère que les propriétés globales des objets qu'il étudie.

Un demi-siècle après al-Khayyãm, son successeur, al-Ṭusī, s'impose une contrainte supplémentaire : démontrer l'existence du point d'intersection de deux courbes dont l'abscisse détermine la racine positive demandée. C'est donc tout naturellement que le mathématicien fut conduit à poser les problèmes de localisation et de séparation des racines, et à traiter des conditions de leur existence ; problèmes pour la solution desquels il fut amené à définir la notion de maximum d'une expression algébrique, et à s'efforcer de trouver concepts et méthodes pour la détermination des maxima. Non seulement cette démarche entraîna al-Túsí jusqu'à l'invention de notions et de méthodes qui ne furent baptisées que plus tard - telle la dérivée - mais elle lui imposa de changer le mode d'approche : pour la première fois, que je sache, il découvre la nécessité de procéder localement.

Tous ces résultats obtenus par al-Ṭusi, ainsi que la théorie qui les englobe, sont d'une évidente importance, et souvent attribués à des mathématiciens postérieurs de plusieurs siècles. Pour mieux dégager la démar- 
che d'al-Ṭüsí, considérons, à propos d'un exemple, les problèmes soulevés et résolus. Soit l'équation

cette équation se réécrit

$$
a x^{2}=x^{3}+c ;
$$

$$
c=x^{2}(a-x) .
$$

Posons

(2)

Al-Ṭüsĩ considère ces trois cas :

$$
f(x)=x^{2}(a-x) \text {. }
$$

c $>\frac{4 \mathrm{a}^{3}}{27}$, le problème est impossible, selon al-Túsī (admet une solution $c=\frac{4 a^{3}}{27}, \begin{aligned} & \text { al-Túsi détermine la racine double } x_{0}=\frac{2 a}{3} \text { (mais ne recon- } \\ & \text { naît pas la racine négative); }\end{aligned}$ $c<\frac{4 \mathrm{a}^{3}}{27}$, al-Ṭusĩ détermine les deux racines positives, avec

$$
0<\mathrm{x}_{1}<\frac{2 \mathrm{a}}{3}<\mathrm{x}_{2}<\mathrm{a} \text {. }
$$

Al-Ṭusĩ étudie le maximum de (2); il montre

$$
\mathrm{f}\left(\mathrm{x}_{\mathrm{o}}\right)=\sup _{0<\mathrm{x}<\mathrm{a}} \mathrm{f}(\mathrm{x}) \quad \text { avec } \mathrm{x}_{\mathrm{o}}=\frac{2 \mathrm{a}}{3} .
$$

Pour trouver $x_{0}=\frac{2 a}{3}$, al-Tínsi résout

$$
f^{\prime}(x)=0 \text {. }
$$

Al-Ṭúsì calcule le maximum de (2)

$$
f\left(x_{o}\right)=f\left(\frac{2 a}{3}\right)=\frac{4 a^{3}}{27},
$$

ce qui permet de justifier les trois cas considérés précédemment. Pour déterminer la racine $x_{2}$, al-Ṭüsi pose

l'équation

$$
\mathrm{x}_{2}=\mathrm{x}_{\mathrm{o}}+\mathrm{X} \text {; cette transformation affine conduit à }
$$

$$
\mathrm{X}^{3}+\mathrm{a} \mathrm{X}^{2}=\mathrm{k},
$$

avec $k=c_{o}-c=\frac{4 a^{3}}{27}-c$, équation déjà résolue par al-Ṭūisi. Il justifie ensuite la transformation affine.

Pour déterminer l'autre racine $x_{1}$, al-Tiusi procède également par la transformation affine $x_{1}=X+a-x_{2}$; avec $X$ la solution positive d'une équation précédemment résolue dans le Traité des équations ${ }^{5}$.

5. Cf. R. RASHEd, éd., Sharaf al-Din al-Tüsi, Qeuvres mathématiques. Algèbre et géométrie au XII' siècle, t. I et II, Paris, Les Belles Lettres, 1986. 
Al-T üsi montre ensuite que $x_{1} \neq x_{0}$ et $x_{1} \neq x_{2}$, et justifie la transformation affine.

Tels sont donc les principaux aspects de cette dialectique entre l'algèbre et la géométrie. Il faudrait encore évoquer, pour être complet, les deux obstacles qui ont ralenti la progression de ces nouvelles mathématiques, en freinant notamment l'expansion de l'analyse locale : le manque d'audace dans l'usage des nombres négatifs comme tels, alors qu'ils n'étaient pas encore définis, et la faiblesse du symbolisme. Autant de questions qui précisément deviendront par la suite objet de préoccupation pour les mathématiciens.

Si l'on souscrit à l'analyse précédente, alors rien ne permet de classer en des ères distinctes les travaux qui ont vu le jour à partir du IXe siècle, et ceux qui furent accomplis plus tard, jusqu'au début du XVII siècle. Tout suggère en effet qu'il s'agit là globalement de la même mathématique. Il suffit pour s'en convaincre de se livrer à quelques comparaisons, entre les écrits d'al-Samaw'al, par exemple, en algèbre et en calcul numérique, et ceux de Simon Stevin ; entre les résultats d'al-Färisĩ en théorie des nombres et ceux de Descartes ; entre les méthodes d'al-Ṭüsi pour la résolution numérique des équations et celle de Viète ; entre sa recherche des maxima et celle de Fermat ; entre les travaux d'al-Khàzin sur l'analyse diophantienne entière et ceux de Bachet de Méziriac, etc.'Si, d'autre part, nous faisons abstraction des travaux d'al-Khwārizmì, d'Abū Kāmil, d'al-Karaji, entre autres, comment comprendre non seulement l'œuvre de Léonard de Pise et celle des mathématiciens italiens, mais aussi les mathématiques plus tardives du XVII siècle?

La rupture avec cette mathématique n'a pas été nécessairement soudaine, et elle ne s'est pas opérée simultanément dans toutes les disciplines. D'autre part, les lignes de clivage contournent rarement les auteurs, mais traversent souvent leurs œuvres. C'est ainsi que la nouvelle théorie des nombres ne commence pas, comme on a pu l'affirmer, avec l'emploi des méthodes algébriques par Descartes et Fermat, lesquels, en procédant ainsi, ne faisaient que retrouver les résultats d'al-Färisi. Bien au contraire, on peut saisir les débuts de cette nouvelle théorie dans l'application des méthodes purement arithmétiques, c'est-à-dire lorsque, vers 1640 , Fermat invente la « descente infinie » et entreprend l'étude de certaines formes quadratiques. C'est donc au sein même de l'œuvre de Fermat, et aux environs de 1640, qu'a lieu cette rupture. Il en va tout autrement pour le chapitre sur la construction géométrique des équations, entamé par al-Khayyäm, poursuivi par al-Ṭüsī, enrichi par Descartes, et repris par bien d'autres mathématiciens jusqu'à la fin du siècle, voire jusqu'au milieu du siècle suivant.

Il est manifeste que les frontières entre ces différentes périodes sont 
floues et s'enchevêtrent, et que, d'ailleurs, les époques nettement définies n'existent, selon l'expression d'Alexandre Koyré, "que dans les manuels scolaires ». C'est à partir de la première moitié du XVII' siècle que se trament les enchevêtrements et que se localisent les principales ruptures.

L'analyse historique et épistémologique qui, bien entendu, prend en compte les mathématiques arabes ainsi que l'usage qui en était fait en latin, aboutit à l'élaboration d'une configuration mathématique cohérente entre le IXe siècle et les débuts du XVII siècle. Mais le découpage de cette configuration, et donc de cette période, ne peut s'accommoder du cadre de cette dichotomie entre " médiéval » et " moderne ». Celleci apparaît finalement comme un emprunt inadéquat à l'histoire politique, laquelle est doublement décalée par rapport à l'histoire des mathématiques. Le seul découpage qui tienne compte des faits historiques euxmêmes ne peut être que différentiel.

Roshdi RASHED, REHSEIS - C.N.R.S. 